\title{
Competências dos enfermeiros nos serviços hospitalares para gestão em saúde efetiva
}

\section{no contexto de pandemia}

\author{
Competences of nurses in hospital services for effective health management in the context of a \\ pandemic
}

Competencias de las enfermeras de los servicios hospitalarios para una gestión sanitaria eficaz en el contexto de una pandemia

Recebido: 13/08/2021 | Revisado: 18/08/2021 | Aceito: 20/08/2021 | Publicado: 22/08/2021

Bruna Eduarda Lopes Felice

ORCID: https://orcid.org/0000-0003-0596-4485 Faculdade de Medicina de São José do Rio Preto, Brasil E-mail: brunafelice4@gmail.com

Alexandre Lins Werneck

ORCID: https://orcid.org/0000-0002-2911-8091 Faculdade de Medicina de São José do Rio Preto, Brasil E-mail: alexandre.werneck@famerp.br Daise Lais Machado Ferreira

ORCID: https://orcid.org/0000-0003-4747-5364 Faculdade de Medicina de São José do Rio Preto, Brasil E-mail: daise_lais@yahoo.com.br

\begin{abstract}
Resumo
Introdução: Diante às diversas atribuições destinadas aos enfermeiros, somado as intensificações no processo de trabalho decorrentes do contexto pandêmico. É imprescindível o aperfeiçoamento das competências, visando colaborar para assistência e gestão de qualidade. Objetivo:Reconhecer as Competências dos Enfermeiros para Gestão em saúde efetiva nos serviços hospitalares em contexto pandêmico. Métodos: Estudo aprovado pelo Comitê de Ética em Pesquisa daFAMERP, Parecer $\mathrm{n}^{\circ}$ 4027756, de 14/05/2020. Estudo transversal com delineamento descritivo, abordagem quantitativa do tipo analítica. Foi aplicado questionário on-line,composto pela caracterização, avaliação e ordenação do grau de importância das competências e autoavaliação. Foram incluídosenfermeiros atuantes no hospital nas áreasassistencial, clínica e gerencialhá mais de três meses, e excluídos, os enfermeiros residentes e que estavam de fériase licenças maternidade ou médica. Resultados:De acordo com Avaliação das competências, algumas se destacaram negativamente, como "Executar atividades burocráticas", "Ética e Moral", "Saber ouvir", "Paciência", "Seguir Normas da Instituição", "Registro correto no prontuário", "Relatar todas as intercorrências", "Sistematização da Assistência de Enfermagem", "Desenvolvimento de pesquisa", "Conhecimento tecnológico" e "Capacitação e desenvolvimento profissional". Conclusão: O estudo contribuiu para uma visão ampliada sobre a importância das competências no processo de trabalho do Enfermeiro para gestão em saúde efetiva.
\end{abstract}

Palavras-chave: Gestão em saúde; Perfil de competências do enfermeiro; Serviços hospitalares; Pandemia.

\begin{abstract}
Introduction: Given the various attributions assigned to nurses, the intensification in the work process arose from the pandemic context. It is essential to improve skills, aiming to collaborate for assistance and quality management. Objective: Recognize the Competencies of Nurses for Effective Health Management in hospital services in a pandemic context. Methods: Study approved by FAMERP Research Ethics Committee, Opinion $\mathrm{n}^{\circ} 4027756$, of 05/14/2020. Cross-sectional study with descriptive design, quantitative analytical approach. Online questionnaire applied, consisting of characterization, assessment, and ranking of the importance of the competencies and selfassessment. We included nurses working in the hospital in the following three main areas: care, clinical, and managerial for more than three months, and we excluded resident nurses and those on vacation, maternity leave, or medical leave. Results: According to the Competencies Assessment, some that stood out negatively were: Performing bureaucratic activities, Ethics, and morals, know how to listen to the patients, Patience, Following the Institution's Rules, Correct record in the medical record, Reporting all complications, Nursing Care Systematization, Research development, Technological knowledge and Training, and professional development. Conclusion: The study contributes to an expanded view on the importance of skills in the work process of nurses for effective health management.
\end{abstract}

Keywords: Health management; Nurse skills profile; Hospital services; Pandemic. 


\begin{abstract}
Resumen
Introducción: Dadas las diversas atribuciones asignadas al enfermero, la intensificación en el proceso de trabajo surgió del contexto pandémico. Es fundamental mejorar las habilidades, con el objetivo de colaborar para la asistencia y la gestión de la calidad. Objetivo: Reconocer las competencias de las enfermeras para la gestión eficaz de la salud en los servicios hospitalarios en un contexto de pandemia. Métodos: Estudio aprobado por el Comité de Ética en Investigación de la FAMERP, Dictamen $n^{\circ}$ 4027756, del 14/05/2020. Estudio transversal con diseño descriptivo, abordaje analítico cuantitativo. Cuestionario online aplicado, consistente en caracterización, valoración y ranking de la importancia de las competencias y autoevaluación. Se incluyeron enfermeras que trabajaban en el hospital en las siguientes tres áreas principales: asistencial, clínica y gerencial durante más de tres meses, y se excluyeron las enfermeras residentes y las de vacaciones, licencia por maternidad o licencia médica. Resultados: De acuerdo con la Evaluación de Competencias, algunas que se destacaron negativamente fueron: Realizar actividades burocráticas, Ética y moral, Saber escuchar a los pacientes, Paciencia, Seguir las Normas de la Institución, Registro correcto en la historia clínica, Reporte de todas las complicaciones, Sistematización de la atención de enfermería, desarrollo de la investigación, conocimiento y formación tecnológica y desarrollo profesional. Conclusión: el estudio contribuye a ampliar la visión sobre la importancia de las habilidades en el proceso de trabajo del enfermero para una gestión eficaz de la salud.
\end{abstract}

Palabras clave: Gestión em salud; Perfil de habilidades de enfermería; Servicios hospitalarios; Pandemia.

\title{
1. Introdução
}

Diante do intenso processo de globalização associado a avanços tecnológicos, industriais e científicos, há modificações constantes nos diversos setores da sociedade, dentre eles a esfera saúde, fazendo-se necessário as adaptações por parte dos profissionais da saúde (Dias, Toledo, Amaro, \&Siman, 2017). Além da imprescindibilidade na qualificação e aprimoramento, a fim de desenvolver os conhecimentos, habilidades e atitudes inerentes aos serviços de saúde (Oliveira, Silvino, Christovam, \& Vidigal, 2016; Resolução CNE/CES n. 3, 2001).

As instituições hospitalares carecem de processos de trabalho eficazes e de qualidade, devido a sua alta complexidade. Tornando-se impreterível a escolha por profissionais que possuam perfis de competências e que estejam dispostos a desenvolvê-las. A fim de viabilizar a qualificação na assistência prestada e gestão com primazia (Brown, \&Crookes, 2016). Pois, as competências podem repercutir de modo significativo nos resultados almejados (Henrique Camelo, Rossi Rocha, Pedreschi Chaves, Santos Silva, \& Inácio Soares,2016).

Em ênfase no processo de trabalho do Enfermeiro atuante no âmbito hospitalar, deve-se enfatizar a indispensabilidade de comprometimento com suas atribuições técnicas, sem prejuízo do componente gerencial pertinente a esses profissionais (Holanda, Marra, \& Cunha, 2018). Percebe-se que as funções destinadas aos enfermeiros são exclusivas e amplas (Thoferhn, 2016), visto que exerce papéis de assistência, educação em saúde e gestão (Rocha, \&Munari, 2013). Diante a amplitude de afazeres que compete ao enfermeiro, faz-se necessário a melhoria e aperfeiçoamento das competências para atingir primor nas atividades desenvolvidas e assistência eficiente, consequentemente auxiliando para uma gestão satisfatória (Holandaet al., 2018; Dias, \& Moniz, 2019).

O delineamento das competências para as práticas dos enfermeiros surgiu em 2003 por um acordo do 'ICN Framework ofCompetencies for theGeneralist Nurse", em que estabelecem como base as relações enfermeiropaciente/família/comunidade, viabilizando o cuidado interdisciplinar, tomada de decisão com fundamentação científica, segurança do paciente, humanização e entre outros (Regulamento nº 190, 2015; Queirós, Vidinha, \& Almeida Filho, 2014). Juntamente a isto, as Diretrizes Curriculares Nacionais (DCN) estabelecem um complexo de competências relevantes para as práticas profissionais do enfermeiro, sendo elas: atenção à saúde, tomada de decisão, comunicação, liderança, administração e gerenciamento e educação permanente (Resolução CNE/CES n. 3, 2001).

Ressalta-se que há diversos obstáculos que dificultam o desenvolvimento dos conhecimentos, habilidades e atitudes (Thoferhn, 2016), assim compreender e aperfeiçoar as competências, principalmente nos serviços hospitalares, torna-se fator de dificuldade dentre os profissionais da saúde (Henriques, Soares, \& Leal, 2018). Por outro lado, é importante ressaltar que 
diante do contexto atual da pandemia, frente a tantas dificuldades e desafios os enfermeiros estão manifestando com maior intensidade suas competências, oferecendo notoriedade da importância do mesmo nos serviços de saúde. Deste modo, frente a momentos de extrema incerteza e mudanças diárias, cabe ao enfermeiro propor novas possibilidades de gestão, partindo dos pressupostos primordiais as práticas e relações, como a empatia, cooperação e confiança (Castelo Branco, 2020).

Além disso, salienta-se que em virtude da pandemia, os enfermeiros estão atuando em condições bastante precárias, representando riscos constantes a essa categoria. Porém, mesmo com as dificuldades encontradas na utilização dos conhecimentos, habilidades e atitudes, que são necessárias em todas as fases do cuidado, os enfermeiros possuem importância ainda maior no atual contexto. Assim, além de fortalecerem as suas competências, os enfermeiros auxiliam de forma significativa no combate à pandemia (Jackson et al., 2020). Portanto, favorece a auto avaliação e aconsciência crítica, propiciando ao enfermeiro tornar-se um indivíduo ativo (Coutoet al., 2016).

Assim, diante das diversas atribuições assistenciais e gerenciais destinadas aos enfermeiros, somadas às intensificações no processo de trabalho, decorrentes do contexto atual de pandemia, é imprescindível o desenvolvimento e aperfeiçoamento dos conhecimentos, habilidades e atitudes, visando colaborar para uma assistência e gestão efetivas e de qualidade, além de propiciar a melhoria das relações entre usuário/enfermeiro e equipe/enfermeiro. Visto que, no cenário presente as relações apresentam-se extremamente fragilizadas e os serviços de saúde com diversas lacunas e vulnerabilidades. Por fim, ressalta-se que a ausência de determinadas competências dos enfermeiros pode refletir negativamente na equipe liderada e nas atribuições inerentes a esses profissionais. Assim, viabilizando limitações no alcance de metas e objetivos estabelecidos pela instituição. Dessa forma, objetivo deste estudo é abordar as competências dos enfermeiros no serviço hospitalar em contexto de pandemia da Covid-19, visando identificar de que modo as competências auxiliam e refletem positivamente para uma gestão efetiva e qualidade nas atividades desenvolvidas por enfermeiros.

\section{Metodologia}

Estudo transversal com delineamento descritivo, abordagem quantitativa do tipo analítica com correlação entre variáveis. No método quantitativo, a coleta de dados quantitativos ou numéricos é por meio do uso de medições de grandezas e é possível obter por meio da metrologia, números com suas respectivas unidades. Com isso, geram conjuntos de dados que podem ser analisados por meio de técnicas matemáticas (Pereira A. S. et al., 2018).

Estudo aprovado pelo Comitê de Ética em Pesquisa da Faculdade de Medicina de São José do Rio Preto (FAMERP), Parecer $n^{\circ} 4027756$, de 14/05/2020. Todos os participantes assinaram o Termo de Consentimento Livre Esclarecido, conforme exige a Resolução no. 466, de 12 de dezembro de 2012, do Conselho Nacional de Saúde, que rege as pesquisas envolvendo seres humanos. Assim como obedeceu a todos os preceitos éticos legais, que envolve as pesquisas com seres humanos.

A pesquisa foi desenvolvida em um hospital de referência, situado no Estado de São Paulo, sendo aplicado um questionário on-line nos diversos setores do hospital. A população foi composta pelos enfermeiros atuantes no contexto hospitalar, dentre eles os assistenciais, clínicos e gerenciais. Foi realizada uma amostragem não probabilística em sequência, ou seja, participaram desta investigação todos os enfermeiros elegíveis atuantes no hospital a mais de três meses e ativos na área assistencial, clínica e gerencial, no período de novembro de 2020 a fevereiro de 2021. Esse período de trabalho se justifica pelo fato de ter passado a experiência profissional. Critérios de exclusão foram os enfermeiros residentes e aqueles que estiverem de férias, licença maternidade e licença médica.

$\mathrm{Na}$ amostragem não probabilística, os pesquisadores elegem elementos por métodos não aleatórios nos quais cada elemento não tem a chance de ser incluído. A amostra não probabilística, em comparação com a probabilística, é menos propensa a produzir amostras representativas - embora a maioria das pesquisas em enfermagem e em outras áreas seja composta por amostras não probabilística. Quando os pesquisadores estudam o efeito de uma causa que eles não podem 
manipular, realizam pesquisa correlacional para examinar as relações entre as variáveis. Correlação é uma associação entre duas variáveis, ou seja, uma tendência de oscilação em uma das variáveis está relacionada com uma variação na outra (p. ex., o peso e a altura das pessoas). As correlações podem ser detectadas por análises estatísticas (Polit, Denise F., 2019).

Os enfermeiros responderam a um questionário com 39 perguntas, constituído por três partes: parte (1) destinou-se a caracterizar o perfil dos enfermeiros respondentes; parte (2) avaliou do grau de importância das competências e ordenação por grau de importância dos grupos de competências (validação estatística) e a parte (3) constituída da autoavaliação. A caracterização destina-se a conhecer a função desempenhada na instituição (Gerência, Assistência, Técnico), sexo, idade e tempo de trabalho no hospital.

A validação estatística foi realizada por meio de escala de avaliação, que permite ao respondente avaliar a descrição das competências e graus de importância (Sem importância, pouco importante, Indiferente, Importante, Muito importante). Na autoavaliação, os respondentes avaliaram o seu próprio desempenho, tendo como parâmetro a descrição das competências, numa escala numérica de níveis que variam do nível (1) ao Nível (5). O Nível (1) Representa: Não desempenha; Nível (2) Representa: Desempenha Pouco; Nível (3) Representa: Desempenha medianamente; Nível (4) Representa: Alto Desempenho; Nível (5): Desempenho excelente.

No questionário para Enfermeiros, estes responderam a 64 questões, indicando a importância que cada competência tem para si, numa escala numérica de níveis que variam do nível (1) ao (5). Sendo 1 = Sem importância; $2=$ Pouco importante; 3 = Importante; 4 = Muito importante; 5 = extremamente importante. O mapeamento buscou identificar o grau de atendimento, a partir da autoavaliação dos enfermeiros efetivos no hospital, em relação às competências descritas. Na coleta de dados relacionada às competências, foram aplicados questionários para confirmar a importância daquelas identificadas.

A pesquisadora foi aos locais de coleta para informar aos participantes os motivos da pesquisa. O instrumento de coleta foi construído na plataforma Formulários Google ${ }^{\circledR}$ e divulgado via internet, por meio dos aplicativos e redes sociais, como WhatsApp, Instagram e Facebook, e e-mail, entre os meses de novembro de 2020 a fevereiro de 2021. A utilização da internet, como recurso auxiliou a troca e disseminação de informações, possibilitou a melhoria e a agilidade do processo de pesquisa. Além de permitir ao pesquisador o contato rápido e preciso com os indivíduos participantes do estudo. O ambiente virtual proporciona, de forma flexível e dinâmica, a formação de redes de pessoas que compartilham ideias e experiências em comum. A utilização de ferramentas virtuais também possibilita a dispersão das informações e divulgação de pesquisas realizadas na área de enfermagem, tornando-se um recurso para a promoção e desenvolvimento da profissão. O Termo de Consentimento Livre e Esclarecido (TCLE) está no corpo do questionário online, com opção para aceitação ou não da participação na pesquisa (Apêndice B).

Após a tabulação dos dados coletados, foram exercidas duas funções de análises estatísticas: descritiva e inferencial. De maneira descritiva, foi traçado o perfil da amostra estudada, contemplando as variáveis analisadas e seus desdobramentos. Os dados foram replicados de forma absoluta e relativa, nesta primeira parte. No âmbito inferencial, foi traçado como objetivo estatístico, a análise de *dependência* e predição entre as variáveis propostas no escopo do trabalho. Foi executado o teste de normalidade de KolmogorovSmirnov.Os resultados *da dependência* entre as variáveis propostas foram obtidos por meio de análise entre os valores de P (significância). Todas as análises foram obtidas por meio do software SPSS Statistics ${ }^{\circledR}$ (versão 23), atrelado às funcionalidades da ferramenta Microsoft Excel® (versão 2016). Para descrição dos resultados, foram calculadas as frequências relativas e absolutas, média, desvio padrão e mediana. Para conhecer os desfechos das variáveis não paramétricas foi aplicado o teste U de Mann-Whitney. O resultado foi considerado significativo quando $\mathrm{p}<0,05$.

Foi analisada a parte inferencial dos cruzamentos estatísticos, nos quais o método de análise se deu por meio do resultado do teste de normalidade da variável. Os métodos escolhidos para abordagem das análises de variação dos resultados entre os grupos analisados,visaram verificar a relação entre eles, nos quais parametrizamos uma das variáveis como sendo 
DEPENDENTE e a outra como INDEPENDENTE, objetivando a análise de predição entre ambas.Lembrando que em todas as análises feitas, o resultado foi obtido para um valor de $\mathrm{p}<0,05$, o que caracterizou significância entre os grupos estudados.Todos os testes contemplaram um erro alfa de $5 \%$ e confiabilidade de $95 \%$.Em todas os cruzamentos, foram aplicados o teste de Kolmogorov-Sminorv de distribuição diferente de Normal.

\section{Resultados}

A amostra desta pesquisa foi constituída na sua grande maioria por mulheres, com idade entre 25 e 35 anos e que trabalham na instituição há 5 anos, como enfermeiras clínicas (Tabela 1).

Tabela 1. Caracterização dos profissionais da pesquisa. São José do Rio Preto, SP, Brasil. 2020.

\begin{tabular}{|c|c|c|}
\hline Sexo & $\mathbf{N}$ & $\%$ \\
\hline Feminino & 46 & 90,20 \\
\hline Masculino & 5 & 9,80 \\
\hline \multicolumn{3}{|l|}{ Idade } \\
\hline 25 a 30 anos & 21 & 41,18 \\
\hline 31 a 35 anos & 21 & 41,18 \\
\hline 36 a 40 anos & 5 & 9,80 \\
\hline 41 a 45 anos & 2 & 3,92 \\
\hline 46 a 50 anos & 2 & 3,92 \\
\hline \multicolumn{3}{|c|}{ Tempo em que trabalha nessa instituição } \\
\hline Até 5 anos & 32 & 62,75 \\
\hline 6 a 10 anos & 11 & 21,57 \\
\hline 11 a 15 anos & 5 & 9,80 \\
\hline 16 a 20 anos & 1 & 1,96 \\
\hline 21 a 25 anos & 2 & 3,92 \\
\hline \multicolumn{3}{|c|}{ No hospital você exerce função } \\
\hline Assistencial & 12 & 23,53 \\
\hline Gerencial & 3 & 5,88 \\
\hline Clínico & 36 & 70,59 \\
\hline TOTAL & 51 & 100,00 \\
\hline
\end{tabular}

Foram retiradas da Tabela, as informações relacionadas à Idade e Tempo que em trabalha nessa instituição, com valores iguais a zero. Fonte: Autores (2020).

Quadro 2. Avaliação das competências e níveis de importância. São José do Rio Preto, SP, Brasil. 2020. 
Research, Society and Development, v. 10, n. 11, e57101119393, 2021

(CC BY 4.0) | ISSN 2525-3409 | DOI: http://dx.doi.org/10.33448/rsd-v10i11.19393

\begin{tabular}{|c|c|c|c|c|c|}
\hline Competências & Sem importância & $\begin{array}{c}\text { Pouco } \\
\text { importante }\end{array}$ & Importante & $\begin{array}{c}\text { Muito } \\
\text { importante }\end{array}$ & $\begin{array}{c}\text { Extremamente } \\
\text { importante }\end{array}$ \\
\hline Comunicação & $0,00 \%$ & $0,00 \%$ & $3,92 \%$ & $21,57 \%$ & $74,51 \%$ \\
\hline Liderança & $0,00 \%$ & $0,00 \%$ & $7,84 \%$ & $23,53 \%$ & $68,63 \%$ \\
\hline Delegação & $0,00 \%$ & $0,00 \%$ & $19,61 \%$ & $45,10 \%$ & $35,29 \%$ \\
\hline Autoconfiança & $0,00 \%$ & $3,92 \%$ & $13,73 \%$ & $33,33 \%$ & $49,02 \%$ \\
\hline Autonomia & $0,00 \%$ & $0,00 \%$ & $19,61 \%$ & $31,37 \%$ & $49,02 \%$ \\
\hline Responsabilidade & $0,00 \%$ & $0,00 \%$ & $5,88 \%$ & $9,80 \%$ & $84,31 \%$ \\
\hline Pontualidade & $0,00 \%$ & $0,00 \%$ & $13,73 \%$ & $21,57 \%$ & $64,71 \%$ \\
\hline Organização & $0,00 \%$ & $0,00 \%$ & $9,80 \%$ & $31,37 \%$ & $58,82 \%$ \\
\hline Paciência & $0,00 \%$ & $3,92 \%$ & $17,65 \%$ & $25,49 \%$ & $52,94 \%$ \\
\hline Ética e moral & $0,00 \%$ & $0,00 \%$ & $5,88 \%$ & $11,76 \%$ & $82,35 \%$ \\
\hline Saber ouvir/escutar & $0,00 \%$ & $0,00 \%$ & $7,84 \%$ & $19,61 \%$ & $72,55 \%$ \\
\hline Conhecimento técnico & $0,00 \%$ & $0,00 \%$ & $11,76 \%$ & $17,65 \%$ & $70,59 \%$ \\
\hline $\begin{array}{l}\text { Desenvolvimento de } \\
\text { pesquisa }\end{array}$ & $0,00 \%$ & $7,84 \%$ & $52,94 \%$ & $23,53 \%$ & $15,69 \%$ \\
\hline $\begin{array}{l}\text { Ajuste/ Adaptação } \\
\text { profissional }\end{array}$ & $0,00 \%$ & $1,96 \%$ & $27,45 \%$ & $43,14 \%$ & $27,45 \%$ \\
\hline $\begin{array}{l}\text { Conhecimento } \\
\text { Tecnológico }\end{array}$ & $0,00 \%$ & $7,84 \%$ & $25,49 \%$ & $37,25 \%$ & $29,41 \%$ \\
\hline $\begin{array}{l}\text { Capacitação e } \\
\text { desenvolvimento } \\
\text { profissional }\end{array}$ & $0,00 \%$ & $3,92 \%$ & $17,65 \%$ & $33,33 \%$ & $45,10 \%$ \\
\hline $\begin{array}{l}\text { Seguir normas da } \\
\text { instituição }\end{array}$ & $0,00 \%$ & $1,96 \%$ & $15,69 \%$ & $25,49 \%$ & $56,86 \%$ \\
\hline Trabalho em Equipe & $0,00 \%$ & $0,00 \%$ & $1,96 \%$ & $27,45 \%$ & $70,59 \%$ \\
\hline Educação em saúde & $0,00 \%$ & $0,00 \%$ & $13,73 \%$ & $31,37 \%$ & $54,90 \%$ \\
\hline Esclarecimento de & $0,00 \%$ & $0,00 \%$ & $21,57 \%$ & $21,57 \%$ & $56,86 \%$ \\
\hline
\end{tabular}




\begin{tabular}{|l|c|c|c|c|c|}
\hline dúvidas dos clientes & & & & \\
\hline $\begin{array}{l}\text { Esclarecimento de } \\
\text { dúvidas da equipe }\end{array}$ & $0,00 \%$ & $0,00 \%$ & $11,76 \%$ & $23,53 \%$ & $64,71 \%$ \\
\hline $\begin{array}{l}\text { Sistematização da } \\
\text { assistência de } \\
\text { enfermagem }\end{array}$ & $0,00 \%$ & $0,00 \%$ & $5,88 \%$ & $33,33 \%$ & $60,78 \%$ \\
\hline $\begin{array}{l}\text { Registro correto no } \\
\text { prontuário }\end{array}$ & $0,00 \%$ & $0,00 \%$ & $11,76 \%$ & $17,65 \%$ & $70,59 \%$ \\
\hline $\begin{array}{l}\text { Receber e passar plantão } \\
\text { Executar todos os } \\
\text { cuidados prescritos }\end{array}$ & $0,00 \%$ & $0,00 \%$ & $9,80 \%$ & $21,57 \%$ & $68,63 \%$ \\
\hline $\begin{array}{l}\text { Relatar todas as } \\
\text { intercorrências }\end{array}$ & $0,00 \%$ & $0,00 \%$ & $5,88 \%$ & $21,57 \%$ & $72,55 \%$ \\
\hline $\begin{array}{l}\text { Executar atividades } \\
\text { burocráticas }\end{array}$ & $0,00 \%$ & $1,96 \%$ & $5,88 \%$ & $11,76 \%$ & $80,39 \%$ \\
\hline $\begin{array}{l}\text { Visão clínica ampliada } \\
\text { Conhecimento teórico }\end{array}$ & $0,00 \%$ & $1,96 \%$ & $7,84 \%$ & $35,29 \%$ & $54,90 \%$ \\
\hline
\end{tabular}

Foram retiradas da Tabela, as informações de menor significância de resultados. Fonte: Autores (2020).

Nos cruzamentos inferenciais, a análise se ocorre por meio do teste de normalidade da variável. Os métodos escolhidos para abordagem das análises de variação dos resultados entre os grupos analisados, visam em suma verificar a relação entre eles, em que se parametriza uma das variáveis como sendo DEPENDENTE e a outra como INDEPENDENTE, objetivando a análise de predição entre ambas.

Na variável de Cruzamento: "Avalie a competência 12 com relação ao seu percebido grau de importância” versus "Atuar considerando o código de ética no exercício de qualquer atividade, no ambiente de trabalho", percebemos que as variáveis analisadas a partir do método de Mann-Whitney, ESTÃO estatisticamente correlacionadas ( $\mathrm{p}=0,014)$. Todos os demais cruzamentos não foram estatisticamente significantes.

\section{Discussão}

Nesta pesquisa, a amostra foi composta por 51 enfermeiros, com prevalência do sexo feminino (90,20\%), o que corrobora um estudo, cuja população foi de 45 enfermeiros e 80\% correspondiam ao sexo feminino (Soareset al., 2019), o que é congruente com outra pesquisa, cuja população era de 84 enfermeiros e 90,5\% eram do sexo feminino (Silva et al., 2017). Essa representatividade pode ser explicada, pois apenas no ano de 1949, em vista a constituição de outras escolas de enfermagem, foi extinta a obrigatoriedade de somente mulheres cursarem a enfermagem (Moreira, 1999). 
Na presente pesquisa, dos 51 enfermeiros, 23,53\% eram assistenciais. Um estudo realizado pela Universidade Federal do Pará (PA) e aplicado no Hospital Universitário João de Barros Barreto, com 44 enfermeiros, apresentou resultados divergentes da atual pesquisa, visto que os enfermeiros assistenciais constituíam 52,27\% (Pontes, 2018). É possível explicar esta discordância, decorrente de populações diferentes, uma vez que a atual pesquisa foi desenvolvida em um hospital localizado no estado de São Paulo, de grande porte, referência para 102 cidades do interior e outros estados e a outra em Belém (PA), um hospital universitário de pequeno porte. Nesta pesquisa, destaca-se maior número de enfermeiros clínicos (70,59\%). Não achamos artigos que corroborassem nossos dados.

Em correlação com os resultados encontrados anteriormente, destaca-se que a competência "Executar atividades burocráticas" foi classificada como pouco importante para 3,92\% dos enfermeiros respondentes e 15,69\% como importante. Em comparação a um estudo qualitativo, com amostra de oito enfermeiros, realizado no Hospital Universitário Antônio Pedro (HUAP) da Universidade Federal Fluminense (UFF), situado na cidade de Niterói (RJ), foi possível perceber sentimentos de frustração e angústia no afastamento dos pacientes, em detrimento dos aspectos burocráticos, interferindo de modo negativo na relação enfermeiro-paciente (Tavares, Gomes, Tavares, \& Passos, 2018). Diante da correlação entre as pesquisas, é perceptível que apesar da divergência entre a forma de coleta de dados, ambas demonstram a necessidade de maior entendimento e valorização das atribuições gerenciais e burocráticas.

A partir dessa evidência, é possível perceber que o histórico da formação de enfermeiras, junto às bases ideológicas, proporcionou a valorização das funções assistenciais e, por outro lado, a desvalorização dos processos gerenciais, visto que, diversos enfermeiros se identificam com atribuições de assistência, pois remete atos de cuidado, modificando a natureza do trabalho. Ressalta-se que os componentes assistenciais e gerenciais são indissociáveis, e que a junção destes, é o que torna o trabalho do enfermeiro singular (Leal, \& Melo, 2018).

Em relação à competência "Ética e moral", na análise inferencial realizada, foi possível identificar que a variável (no hospital você exerce função de) está estatisticamente correlacionada ( $\mathrm{p}=0,014)$ com o percebido grau de importância na competência 12 "Atuar considerando o código de ética no exercício de qualquer atividade, no ambiente de trabalho", ou seja, percebeu-se que a avaliação de importância varia conforme função em que se tem no serviço hospitalar, sendo elas assistencial,gerencial e clínica. Dos enfermeiros, 5,88\% acreditam ser apenas importante a competência "Ética e moral", enquanto $11,76 \%$ acreditam ser muito importante e,82,35\%, consideraram "Extremamente Importante".

Um estudo que abordou a questão de Ética e Moral, mostrou que em uma amostra de 44 enfermeiros, 11,26\%acreditaram ser importante a competência Ética e Moral e, 88,64\%, muito importante (Pontes, 2018). Outro estudo, com uma amostra de 17diretores de enfermagem de hospitais de alta complexidade, públicos, privados e filantrópicos na Região Sul do Brasil, relatou que, em relação à competência "Respeito Ético" e legal da profissão, 100\% alegaram a positividade da competência (Bettiolet al., 2019), dado que diverge de forma significativa desta pesquisa e, pode ser explicado, pela diferença de população, uma vez que a pesquisa atual aplicou o questionário com enfermeiros que exercem funções gerenciais, clínicas e assistenciais e o outro obteve como amostra de diretores de enfermagem.

Salienta-se que as condutas e valores éticos estão descritas no Código de ética Profissional de Enfermagem, viabilizando qualidade nas relações de trabalho (Barbosa, Rodrigues, Celino, \& Costa, 2017). Além disso, a ética se constrói diária e continuadamente no processo de trabalho, de acordo com as experiências com as quais o indivíduo se depara, sendo essencial a criação de uma cultura ética nos vínculos (Germano, 2013). Por outro lado, entende-se que frente ao contexto de pandemia atual, agir de forma ética e moral é um processo que encontra barreiras diante da sobrecarga de trabalho, o que gera desgaste físico e mental (Jacksonet al., 2020).

No que diz respeito à competência "Liderança", ressalta-se que 7,84\% julgaram apenas como importante, 23,53\% como muito importante e $68,63 \%$ como extremamente importante. Em uma pesquisa com enfermeiros, 18,18\% acreditaram ser 
importantes e 79,55\% muito importante (Pontes, 2018). Dessa forma, esses resultados aparecem de forma discrepante com o que se espera, visto que a pesquisa atual foi desenvolvida em um hospital de grande porte, onde há predominância de enfermeiros clínicos, e o outro estudo, desenvolvido em um hospital de pequeno porte, havendo um maior número enfermeiros que desenvolvem função assistencial. E por isto, entende-se que aqueles enfermeiros que possuem função clínica, deveriam ter um maior entendimento, comparado aos enfermeiros assistenciais sobre a importância da Liderança no processo de trabalho e principalmente para a equipe de enfermagem.

É de extrema relevância reforçar o quão importante é essa competência para a prática do enfermeiro, pois a liderança permite que o enfermeiro influencie positivamente sua equipe, contribuindo na melhoria das relações interpessoais, e resultando no maior desempenho nos processos de trabalho, bem como na gestão com excelência (Mondiniet al., 2020). Diante das dificuldades advindas da pandemia nos serviços de saúde, como a superlotação dos leitos, lacunas nos processos de gerir e escassez de insumos, é primordial uma liderança de qualidade dos enfermeiros (Montezeli, Almeida, Hadad, 2018).

Com relação à competência "Comunicação", 3,92\% acreditaram ser apenas importante, 21,72\% muito importante e $74,51 \%$ extremamente importante. Em uma pesquisa com 44 enfermeiros, 22,73\% julgaram importantes e 75,00\% muito importante. Um estudo de abordagem quantitativa, com 152 enfermeiros atuantes em um hospital no Nordeste do Brasil, identificou (Pontes, 2018) correlação entre a comunicação e os sentimentos de prazer e sofrimento no trabalho, ou seja, a partir do momento em que há o aumento de sofrimento no ambiente de trabalho, ocorre diminuição da comunicação, e consequentemente a queda da interação entre os profissionais de enfermagem (Pimentaet al., 2020).

Dessa forma, no cenário da pandemia pela Covid-19, diversas singularidades foram adquiridas como estratégias para amenizar a distância necessária entre familiares e as equipes, bem como profissionais e pacientes, tornando a comunicação um fator imprescindível nessa conjuntura (Ramos, 2020). O enfermeiro possuí papel de relevância no fornecimento de informações para a sua equipe sobre a situação pandêmica, normas instituídas e medidas de prevenção e controle (VenturaSilva et al., 2020).

Com relação à importância que cada indivíduo acreditar ter em relação à competência "Saber ouvir e escutar", 7,84\% responderam como apenas importante e 19,61\% muito importante. Uma pesquisa desenvolvida em uma instituição hospitalar privada, na cidade de Caxias do Sul/SP/Brasil, com 34 profissionais de enfermagem, dos quais 15\% eram enfermeiros, revelou que os profissionais entenderam a relevância do cuidado humanizado, incluindo a empatia e capacidade de ouvir o outro (Luz, \& Souza, 2020). Diante disto, destaca-se que o enfermeiro necessita de olhar crítico e escuta qualificada para desenvolver o cuidado diferenciado a cada indivíduo. A escuta é um elemento de diferenciação do serviço de saúde, o que permite um atendimento adequado e a construção de um plano de cuidado direcionado as principais queixas do paciente (Jasmim, Queluci, Mendonça, Souza, \& Dias, 2018).

Diante da competência "Paciência", 3,92\% avaliaram como pouco importante, o que corrobora uma revisão narrativa realizada em Goiânia (GO), em 2020, que avaliou seis artigos na íntegra, cujo resultado foi a não aplicação da empatia, a falta de atenção, a falta de paciência e a não criação de vínculo entre profissional e usuário (Dourado, 2020). Além disso, um estudo realizado em um Hospital Universitário da Região Sul do país, em duas unidades de clínica cirúrgica, com a participação de 200 profissionais de enfermagem, avaliou a satisfação dos pacientes por diversos itens. Dentre eles,a competência "Os profissionais da equipe de Enfermagem não têm paciência suficiente",mostrou uma média de 4,05, sendo que o valor máximo foi 5,0, resultados que são congruentes com os pontos já citados anteriormente (Santos et al., 2019).

O processo de hospitalização no contexto de pandemia, gera nos pacientes e familiares sentimentos de ansiedade, tristeza e preocupação. Diante disso, faz-se ainda mais necessário a humanização e empatia por parte do enfermeiro e sua equipe durante todo o processo de cuidado (Zhang, Wu, Zhao, \& Zhang, 2020). Por outro lado, é importante salientar que diante de contextos de escassez de insumos, mudanças de rotina, alteração e inovação de protocolos constantes, além da 
sobrecarga (Souzaet al., 2021), em que os enfermeiros se encontram, podem gerar um conjunto de sentimentos e emoções que contribuem como uma lacuna no processo de saber ouvir, escutar e humanização nos diversos serviços.

Com relação à competência, "Seguir normas da instituição", chama a atenção a crença de que é pouco importante, relatado por 1,96\% dos participantes, enquanto 15,69\% caracterizaram apenas como importante. Não foi possível encontrar pesquisas que corroborassem esses dados.

Sobre a competência "Registro correto no prontuário", é importante destacar que 11,76\% responderam importante e $17,65 \%$ responderam muito importante, necessitando um olhar com mais cautela para essa competência. Na competência "Relatar todas as intercorrências" 1,96\% classificaram como pouco importante. Em comparação com uma pesquisa de revisão que identificou 10 artigos selecionados para análise, os resultados mostraram erros de registro dos mais diversos, desde problemas na identificação dos profissionais, relatos incompletos, até ausência de registros importantes, como checagem de medicamentos e materiais (Medeiros, 2020), o que está de acordo com o resultado encontrado na presente pesquisa e nas respostas adquiridas diante da competência "Sistematização da assistência de enfermagem", na qual 5,88\% avaliaram como importante e $33,3 \%$ muito importante.

Um estudo recente mostrou que, de 120 prontuários analisados, foram destacados como inadequabilidade a falta de anotações em alguns dias, a ausência da hora, a identificação do profissional responsável, incompletudes nos registros diários da enfermagem, além de rasuras. Salienta-se que o registro de enfermagem constitui um dever ético-legal a ser realizado por enfermeiros e técnicos de enfermagem em todos os ambientes de saúde (Resolução Cofen n. 311, 2007; Resolução Cofen nº $545,2017)$ e se destaca como ferramenta que documenta e qualifica a assistência prestada. Contudo, mesmo imprescindível e obrigatório, os registros de enfermagem ainda são incipientes, fato que corrobora a invisibilidade da profissão (Maziero, Vannuchi, Haddad, Vituri, \&Tada, 2013).

Em relação à competência "Desenvolvimento de pesquisa", 7,84\% dos enfermeiros julgaram como pouco importante. Em uma pesquisa qualitativa desenvolvida no Hospital Universitário João de Barros Barreto, com 5 enfermeiros, uma enfermeira expressou que essa competência é relevante, porém possuía pouco incentivo e que em virtude da rotina atribulada, podia-se compreender o porquê do baixo desenvolvimento por parte dos enfermeiros (Pontes, 2018). Resultado que corrobora um estudo de abordagem qualitativa, vinculado a um Hospital Universitário, com 25 profissionais de enfermagem, sendo dez enfermeiros assistenciais, seis enfermeiros pesquisadores, além de nove estudantes da graduação em enfermagem, uma enfermeira assistencial pontuou que deveria ocorrer a percepção da necessidade de inovações científicas, porém ela não percebeu isso em sua prática (Silva, Leite, Trevizan, Silva, \& José, 2017).

Ressalta-se que a divergência entre os estudos pode ser explicada pela diferença da população, que nesta pesquisa, contou com a participação de enfermeiros clínicos, assistenciais e gerenciais e, no estudo mencionado, foi realizada entrevista com um enfermeiro gerencial, e na seguinte por um enfermeiro assistencial, o que poderia afetar o resultado. É importante ressaltar que a presente pesquisa foi realizada em um hospital escola. Dessa forma, compreende-se a necessidade de que os enfermeiros entendam a importância do desenvolvimento de pesquisa e participem a fim de agregar mais conhecimento nos setores, e consequentemente produzir benefícios em todos os âmbitos inerentes à saúde.

Salienta-se que há extrema necessidade de fortalecimento entre o âmbito assistencial e o de pesquisa, viabilizando um cuidado com respaldo em evidências científicas, atuais e de qualidade, uma vez que a medicina está em constante evolução e mudança. É importante ressaltar que as instituições motivem a realização de pesquisas, para que os profissionais se aprimorem, agreguem conhecimentoaos locais em que trabalham, para atenderde forma indireta e/ou direta o paciente. No contexto atual de pandemia, a realização de pesquisas torna-se fundamental no processo de trabalho do enfermeiro, mantendo-o atualizado. Fator de extrema importância, frente ao cenário da Covid-19, no qual as informações se alteram com intensa frequência. 
No que diz respeito à competência "Conhecimento tecnológico", 7,84\% acreditaram ser pouco importante. No entanto, não achamos artigos que corroborassem nossos dados. A partir do resultado demonstrado, deve-se ressaltar que essa competência é extremamente importante na prática do enfermeiro, visto que há diversas tecnologias presentes no dia a dia desses profissionais, além de haver outras que estão sendo implantadas e incorporadasao processo de trabalho (tecnologias duras). Sabe-se que as tecnologias leves - caracterizadas pelo vínculo e acolhimento, leve/duras - denominadas pelo conhecimento estruturado e duras - equipamentos e estruturas organizacionais, oferecem um atendimento humanístico e de qualidade (Rossi, \& Lima, 2005), fatores de máxima importância no contexto de pandemia atual.

Por fim, a competência "Capacitação e desenvolvimento profissional”, foi avaliada como pouco importante para 3,92\% da amostra e importante para 17,65\%. Resultado que merece bastante atenção, pois no cenário atual, os enfermeiros são os profissionais que atuam na linha de frente da assistência, representando maior risco de exposição ao vírus da Covid-19, o SARS-CoV-2. Destacando-se como líderes da realização de cuidados com diferentes níveis de complexidade, como promoção em saúde sobre a higienização das mãos, ações complexas do cuidado e entre outras (Viana, 2019; Ramos,2020). Dessa forma, a capacitação dos enfermeiros que estão na linha de frente no combate à pandemia, viabiliza a correta colocação, utilização e remoção de Equipamentos de Proteção Individual (EPIs) (Reis, Lago, Carvalho, Nobre, \& Guimarães, 2020), além de proporcionar maior segurança para a atuação dos enfermeiros, como também para os pacientes que estão recebendo os cuidados prestados.

Salienta-se a relevância de os enfermeiros compreenderem e praticarem os conhecimentos, habilidades e atitudes, uma vez que as competências atuam de modo direto e indireto sob o setor, viabilizando uma gestão efetiva e de qualidade. Além de promoverem a melhoria no cuidado e nas relações entre equipe-enfermeiro e enfermeiro-paciente (Mazzoni, Bittencourt, \& Ribeiro, 2018). Compreende-se que no contexto atual, há intensa sobrecarga e exaustão dos enfermeiros, implicando em desafios diários a serem enfrentados por esses profissionais. Diante de tantos desafios, é importante ressaltar que a assistência prestada e as relações enfermeiro-paciente, enfermeiro-equipe, encontram-se prejudicadas (Liuet al., 2020). Dessa forma, é importante que haja o fortalecimento e oferta de condições apropriadas para a atuação dos enfermeiros nesse contex to crítico, possibilitando o desenvolvimento e aperfeiçoamento das competências necessárias, a fim de agregar nas instituições.

Esta pesquisa encontrou diversas limitações, como a dificuldade da adesão dos enfermeiros para que respondessem o questionário on-line, além da necessidade de realizar a comparação de resultados em contextos diferentes, uma vez que, não foram encontrados estudos que abordassem essa temática no cenário pandêmico, e a realização da pesquisa em contexto da pandemia da Covid-19.

\section{Conclusão}

Os conhecimentos, habilidades e atitudes possuem papel fundamental nas práticas do enfermeiro, e diante do cenário atual de pandemia, as competências tornam-se ainda mais imprescindíveis. Ressalta-se que no cenário presente, há necessidade de práticas que dinamizem o serviço, diante da sobrecarga dos setores de saúde e dos respectivos profissionais. Desse modo, quando as competências são compreendidas e executadas é possível trazer benefícios múltiplos para instituição, promovendo uma gestão efetiva e de qualidade, assim como, proporciona melhora nas relações entre a equipe e os pacientes que usufruem do serviço. O estudo contribui para uma visão ampliada e reflexão sobre a importância das competências no processo de trabalho do Enfermeiro, a fim de desenvolver a autocrítica e propiciar o aperfeiçoamento, viabilizando gestão em saúde de qualidade.

A realização do estudo em contexto pandêmico foi um fator limitante. Desse modo, sugere-se que para trabalhos futuros acerca da temática, seja aplicado o questionário novamente em contexto pós- pandêmico. A fim de possibilitar analisar 
e perceber de que modo a pandemia modificou e está relacionada aos conhecimentos, habilidades e atitudes dos enfermeiros atuantes nos serviços hospitalares.

\section{Referências}

Barbosa, M. L., Rodrigues, H. N. S., Celino, S. D. M., \& Costa, G. M. C. (2017). Nursing professionals' knowledge about the ethics code that governs the profession.Revista Baiana de Enfermagem, 31(4), e21978. http://dx.doi.org/10.18471/rbe.v31i4.21978

Bettiol, C. H. O., Pedro, D. R. C., Pissinati, P. S. C, Rossaneis, M. A., Haddad, M. C. F. L., \&Dellaroza, M. S. G. (2019). Professional competences soughtby managers in the selective process of nurses in hospital institutions.Revista Paranaense de Enfermagem, 2(1), 59-65. Recuperado dehttp://seer.fafiman.br/index.php/REPEN/article/view/555/533

Brown, R. A., \& Crookes, P. A. (2016). What are the 'necessary' skills for a newly graduating RN? Results of an Australian survey.BMC Nursing, 15, 23. https://doi.org/10.1186/s12912-016-0144-8

Castelo Branco, V. R. (2020). Inteligência de mercado: a liderança durante e após covid-19. Rhttp://www3.sumare.edu.br/comunicados/2020/Junho/INTELIG\%C3\%8ANCIA\%20DE\%20MERCADO_A\%20LIDERAN\%C3\%87A\%20DURANTE\%20 E\%20AP\%C3\%93S-COVID19\%20(1).pdf

Couto, T. A., Santos, F. P. A., Rodrigues, V. P., Vilela, A. B. A., Machado, J. C., \& Jesus, A. S. (2016). Health education under perspective of family health teams users. Revista de Enfermagem UFPE On Line, 10(5), 1606-1614. https://periodicos.ufpe.br/revistas/revistaenfermagem/article/view/11156/12674

Dias, A. K. G., Toledo, L. V., Amaro, M. F., \&Siman, A. G. (2017). Perception of nurses regarding their managerial role in the hospital context.Revista de Enfermagem UFPE On Line, 11(5), 2185-2194. https://doi.org/10.5205/1981-8963.2019.240535

Dias, R. M., \& Moniz, M. A. (2019). Nursing managerial aptitudes in the family healthstrategy: perception sof nursing under graduates. Revista de Pesquisa Cuidado é Fundamental, 11(4), 1048-1052. https://doi.org/10.9789/2175-5361.2019.v11i4.1048-1052

Dourado, J. S., Lima, N. P. (2020). Percepção dos usuários das unidades de emergência a cerca da competência e responsabilidades dos enfermeiros: revisão narrativa (Trabalho de Conclusão de Curso). Pontifícia Universidade Católica de Goiás, Goiânia, GO, Brasil. https://repositorio.pucgoias.edu.br/jspui/handle/123456789/148

Germano, R. M. (2013). A pesquisa e os dilemas éticos do trabalho da enfermagem. Revista Brasileira de Enfermagem,66(n.esp.):76-79. https://doi.org/10.1590/S0034-71672013000700010

Henrique Camelo, S. H., Rossi Rocha, F. L., Pedreschi Chaves, L. D., Santos Silva, V. L., \& Inácio Soares, M. (2016). Competências profissionais e estratégias organizacionais de gerentes de enfermagem. Ciencia y Enfermería, 22(1), 75-86. https://doi.org/10.4067/S0717-95532016000100007

Henriques, S. H., Soares, M. I., \& Leal, L. A. (2018). Applicability assessment of the portuguese version of a competency questionnaire for hospital nurses. Texto \& Contexto Enfermagem, 27(3), e2140017. https://doi.org/10.1590/0104-070720180002140017

Holanda, F. L., Marra, C. C., \& Cunha, I. C. K. O. (2018). Assessment of professional competence of nurses in emergencies: created and validated instrument. Revista Brasileira de Enfermagem, 71(4), 1865-1874. http://doi.org/10.1590/0034-7167-2016-0595

Jackson, D., Bradbury-Jones, C., Baptiste, D., Gelling, L., Morin, K., Neville, S., \& Smith, G. D. (2020). Life in the pandemic: some reflections on nursing in the context of COVID-19. JournalofClinicalNursing, 29(13-14), 2041-2043. doi: https://doi.org/10.1111/jocn.15257

Jasmim, J. S., Queluci, G. C., Mendonça, A. R., Souza, V. R., \& Dias, S. F. C. (2018). Competências do enfermeiro na Estratégia Saúde da Família. Revista de Enfermagem UFPE OnLine, 12(11), 2906-2915. 3 https://doi.org/10.5205/1981-8963-v12i11a237846p2906-2915-2018

Leal, J. A. L., \& Melo, C. M. M. (2018). The nurses' workprocess in different countries: an integrative review. Revista Brasileira de Enfermagem, 71(2), 413423. http://doi.org/10.1590/0034-7167-2016-0468

Liu, Q., Luo, D., Haase, J. E., Guo, Q., Wang, X. Q., Liu, S., Xia, L., Liu, Z., Yang, J., \& Yang, B. X. (2020). The experiences of health-care providers during the COVID-19 crisis in China: a qualitative study. Lancet Global Health, 8(6), e790-e798. https://doi.org/10.1016/S2214-109X(20)30204-7

Luz, B. R., \& Souza, J. S. M. (2020). Humanização no centro cirúrgico: percepção da equipe de enfermagem. Revista Expressão Católica Saúde, 5(2), 56-63. http://doi.org/10.25191/recs.v5i2.3760

Maziero, V. G., Vannuchi, M. T. O., Haddad, M. C. L., Vituri, D. W., \& Tada, C. N. (2013). Quality control of patients' monitoring records in a university hospital. REME RevistaMineira de Enfermagem, 17(1): 165-170. https://doi.org/10.5935/1415-2762.20130014

Mazzoni, V. G., Bittencourt, L. P., \& Ribeiro, M. L. (2018).Desafios da dimensão organizacional do cuidado no cotidiano de trabalhadores de enfermagem. Revista Enfermagem UFPE OnLine, 12(1), 11-18. https://doi.org/10.5205/1981-8963-v12i1a230392p11-18-2018

Medeiros, R. P. (2020). Impacto de registros de enfermagem inadequados nas glosas hospitalares (Trabalho de Conclusão de Curso). Escola de Formação Complementar do Exército, Escola de Aperfeiçoamento de Oficiais, Brasília, DF, Brasil. https://bdex.eb.mil.br/jspui/bitstream/123456789/7954/1/CAM_QCO_2020_Cap\%20Rafael\%20Pantuzzo.pdf

Mondini, C. C. S. D., Cunha, I. C. K. O., Trettene, A. S., Fontes, C. M. B, Bachega, M. I., \&Cintra, F. M. R. (2020). Authentic leadership among nursing professionals: knowledge and profile. RevistaBrasileira de Enfermagem, 73(4), e20180888. https://doi.org/10.1590/0034-7167-2018-0888

Montezeli, J. H., Almeida, K. P., Hadad, M. C. F. L. (2018). Nurses' perceptions about social skills in care management from the perspective of complexity. Revista da Escola de Enfermagem da USP,52, e03391. https://doi.org/10.1590/S1980-220X2017048103391 
Moreira, M. C. N. (1999). A Fundação Rockefeller e a construção da identidade profissional de enfermagem no Brasil na Primeira República. História, Ciências, Saúde-Manguinhos, 5(3), 621-645. https://doi.org/10.1590/S0104-59701999000100005

Oliveira, C. D., Silvino, Z. R., Christovam, B. P., \& Vidigal, P. D. (2016). Revisão integrativa sobre a formação do enfermeiro baseada em competências. Revista Baiana de Saúde Pública, 40(1), 9-13. https://doi.org/10.22278/2318-2660.2016.v40.n1.a660

Pimenta, C. J. L., Viana, L. R. C., Bezerra, T. A., Silva, C. R. R., Costa, T. F., \& Costa, K. N. F. M. (2020). Pleasure, suffering and interpersonal communication in the work of nurses in the hospital setting.Texto \& Contexto Enfermagem, 29, e20190039. https://doi.org/10.1590/1980-265X-TCE-20190039

Polit, D. F., \& Beck. C. T. (2019). Fundamentos de Pesquisa em Enfermagem - Avaliação de evidências para a prática da enfermagem (9a. ed.). Porto Alegre: Artmed.

Pontes, D. A. F. S. (2018). Mapeamento de competências dos enfermeiros do hospital universitário João de Barros Barreto (Trabalho de Conclusão de Curso). Universidade Federal Pará, Bo Belém, https://bdm.ufpa.br:8443/jspui/bitstream/prefix/1623/1/TCC_MapeamentoCompetenciaEnfermeiros.pdf

Queirós, P. J. P., Vidinha, T. S., \& Almeida Filho, A. J. (2014). Self-care: Orem's theoretical contribution to thenursing discipline and profession. Revista de Enfermagem Referência, 4(3), 157-163. https://doi.org/10.12707/RIV14081

Ramos, R. S. (2020). Oncology Nursing in Coping with the COVID-19 Pandemic: Reflections and Recommendations for Oncology Care Practice. Revista Brasileira de Cancerologia, 66, e-1007. https://doi.org/10.32635/2176-9745.RBC.2020v66nTemaAtual.1007

Regulamento $n^{\circ}$ 190, de 6 de março de 2015. Regulamento do perfil de competências do enfermeiro de cuidados gerais. https://dre.pt/application/file/a/67058698

Reis, L. M., Lago, P. N., Carvalho, A. H. S., Nobre, V N., \& Guimarães, A. P. (2020). Atuação da enfermagem no cenário da pandemia COVID-19. Nusing (São Paulo), 23(269), 4765-4768. https://doi.org/10.36489/nursing.2020v23i269p4765-4772

Resolução CNE/CES $n^{o}$ 3, de 7 de novembro de 2001. Institui Diretrizes Curriculares Nacionais do Curso de Graduação em Enfermagem. http://portal.mec.gov.br/cne/arquivos/pdf/CES03.pdf

Resolução Cofen $n^{\circ}$ 311/2007. Aprova a reformulação do código de ética dos profissionais de enfermagem. http://www.cofen.gov.br/resoluo-cofen3112007_4345.html

Resolução Cofen $n^{\circ}$ 545/2017. Revoga a Resolução Cofen $n^{\circ}$ 191/1996. Dispõe sobre a anotação de enfermagem e mudança nas siglas das categorias profissionais. http://www.cofen.gov.br/wp-content/uploads/2017/05/RES.-545-17.pdf

Rocha, B. S., \&Munari, D. B. (2013). Avaliação da competência interpessoal de enfermeiros coordenadores de equipe na saúde da família. Revista de Enfermagem e Atenção à Saúde, 2(3), 53-66. Recuperado de http://seer.uftm.edu.br/revistaeletronica/index.php/enfer/article/viewFile/430/434

Rossi, F. R., \& Lima, M. A. D. (2005). Acolhimento: tecnologia leve nos processos gerenciais do enfermeiro. Revista Brasileira de Enfermagem, 58(3), 305310. https://doi.org/10.1590/S0034-71672005000300010

Santos, J. L. G., De-Pin, S. B., Menegon, F. H., Sebold, L., Nascimento, K. C., \&Gelbcke, F. L. (2019). Satisfaction of patients about the nursing care in surgical units: mixed method research. REME Revista Mineira de Enfermagem, 23, e-1229. https://doi.org/10.5935/1415-2762.20190077

Silva, I. R., Leite, J. L., Trevizan, M. A., Silva, T. P., \& José, S. A. P. (2017). Connections between research and health care assistance: Emerging challenges for science, innovation and technology in nursing. Texto \& Contexto Enfermagem, 26(4), e24700166. http:// doi.org/10.1590/0104-07072017002470016

Silva, V. L. S., Camelo, S. H. H., Soares, M. I, Resck, Z. M. R., Chaves, L. D. P., \& Santos, F. C. (2017). Leadershippractices in hospital nursing: a self of manager nurses. Revista da Escola de Enfermagem da USP, 51, e03206. https://doi.org/10.1590/S1980-220X2016099503206

Soares, M. I., Leal, L. A., Resck, Z. M. R., Terra, F. S., Chaves, L. D. P., \& Henriques, S. H. (2019). Avaliação de desempenho por competências em enfermeiros hospitalares. Revista Latinoamericana de Enfermagem, 27, e3184. https://doi.org/10.1590/1518-8345.3173.3184

Souza, N. V. D. O., Carvalho, E. C., Soares, S. S. S., Varella, T. C. M. M. L., Pereira, S. R. M., \& Andrade, K. B. C. (2021). Nursing work in the COVID-19 pandemic and repercussions for workers'mental health.RevistaGaúcha de Enfermagem, 42(spe), e20200225.https://doi.org/10.1590/19831447.2021.20200225

Tavares, M. M., Gomes, A. M. T., Tavares, C. M. M., \& Passos, J. P. (2018). Cotidiano de trabalho do enfermeiro hospitalar: atividade burocrática ou trabalho vivo. Atas - Investigação Qualitativa em Saúde, 2, 605-612. https://proceedings.ciaiq.org/index.php/ciaiq2018/article/view/1826/1778

Thoferhn, M. B. (2016). Enfermagem: manual de gerenciamento. Porto Alegre: Moriá.

Ventura-Silva, J. M. A., Ribeiro, O. M. P. L., Santos, M. R., Faria, A. C. A., Monteiro, M. A. J.,\&Vandresen, L. (2020). Planejamento organizacional no contexto de pandemia por COVID-19: implicações para a gestão em enfermagem. Journal Health NPEPS, 5(1), e4626. http://doi.org/10.30681/252610104626

Viana, R. A. P. P. (2019). Enfermagem e sua atuação: a importância desta nobre profissão. Journal Health NPEPS, 4(2), 14-15. http://dx.doi.org/10.30681/252610104208

Zhang, J., Wu, W., Zhao, X., \& Zhang, W. (2020). Recommended psychological crisis intervention response to the 2019 novel coronavirus pneumonia outbreak in China: a model of West China Hospital.PrecisionClinical Medicine, 3(1), 3-8. http://doi.org/10.1093/pcmedi/pbaa006 